www.ocula.it • ISSN 1724-7810 • Vol 21, No 24 (October 2020) • DOI: 10.12977/ocula2020-47

Quando è design When is Design Quand c'est du design

a cura di Michela Deni e Dario Mangano

\title{
L'apport du design social aux politiques françaises de gestion des risques naturels Contribution à une innovation sociale territorialisée
}

\author{
Béatrice Gisclard \\ PROJEKT (EA 7447) Université de Nîmes, FR
}

\begin{abstract}
Protecting against hazards and organizing disaster management are constant concerns for all human societies. However, current environmental challenges require a rethinking of the division of responsibilities and roles of each individual, both managers and citizens. Starting from the case of France, our research aims to study the social design approach from the perspective of territorialized social innovation, in order to better involve inhabitants in the face of flood risk. We will question the place assigned by the law to the individual as the first actor in his or her security, and then we will detail the conditions for the implementation of a creative workshop. Based on the results obtained, we will study the contribution of design as a factor of individual and collective commitment, as well as its reception by the actors.
\end{abstract}

\section{Keywords}

Natural hazards; Social innovation; Social design; Empowerment; Public policies.

\section{Contents}

o. Introduction

1. Renouveler le rôle du citoyen " acteur de sa sécurité » par le design social ?

2. Expérimenter le risque inondation à travers un atelier créatif

3. Un engagement individuel et collectif par le projet

4. Conclusions

Bibliographie 
Vol 21, No 24 (October 2020) • DOI: 10.12977/ocula2020-47

Béatrice Gisclard | L'apport du design social aux politiques françaises de gestion des risques naturels

\section{o. Introduction}

En France, la loi de Modernisation de la Sécurité Civile du 13 août 2004 a positionné l'individu comme le premier acteur de sa sécurité, introduisant un jalon important dans une reconfiguration des responsabilités face aux risques. Inscrit dans la loi de 2004, l'article $4{ }^{1}$ stipule que :

Toute personne concourt par son comportement à la sécurité civile. En fonction des situations auxquelles elle est confrontée et dans la mesure de ses possibilités, elle veille à prévenir les services de secours et à prendre les premières dispositions nécessaires.

Pourtant, ce changement de paradigme reste encore peu opérant dans les politiques de gestion des risques à l'échelle individuelle. En tant que premier acteur de sa sécurité, l'individu a un rôle à jouer, on attend de lui qu'il ait des comportements adaptés. Or cet article de loi n’apporte pas de précisions sur l'opérationnalisation de ces comportements en cas de situation périlleuse. Le modus operandi de l'État français sur ces sujets est le " porter-à-connaissance » des risques, à travers la réalisation des Plans de Prévention des Risques (PPR) pour ses territoires et la formulation de consignes générales et/ou spécifiques à destination des populations. Mais leur appropriation et leur mise en œuvre par ces dernières restent un sujet largement méconnu et sous-estimé par les institutions. Comme le souligne Bason (2012), la majorité des défis sociaux actuels sont liés aux comportements individuels et collectifs. Le design social, par la place centrale qu'il accorde à l'humain, aux usages, voire aux nouvelles pratiques de l'espace (Jacob et Desage 2015) peut s'avérer un levier majeur pour les affronter, y compris dans le domaine des risques. Cet article propose d'étudier l'opportunité de renouveler le rôle du citoyen « acteur de sa sécurité », via le prisme de l'innovation sociale par le design. À travers une recherche-action située, nous détaillerons la mise en ouvre concrète de cette proposition puis nous analyserons les conditions d'une meilleure appropriation du risque inondation par les habitants à une échelle locale. Nous concluerons sur les perspectives offertes par cette démarche dans les politiques publiques de gestion des risques en soulignant l'apport du design dans ce champ de recherche.

\section{Renouveler le rôle du citoyen² « acteur de sa sécurité » par le design social ?}

1.1. Quels enjeux dans la mise en cuvre effective des consignes comportementales en cas de danger?

La variété des risques encourus (naturels ou anthropiques) correspond à une variété de consignes - se réfugier en hauteur, sortir de son véhicule, éva-

1 Abrogé et remplacé par l'article L721-1 du Code de la sécurité intérieure créé par Ordonnance $\mathrm{n}^{\circ}$ 2012-351 du 12 mars 2012 <https://tinyurl.com/yxjecf4g>.

2 L'usage du masculin dans cet article a pour but d'alléger la forme littéraire et ne saurait avoir d'autre signification. 
Vol 21, No 24 (October 2020) • DOI: 10.12977/ocula2020-47

Béatrice Gisclard | L'apport du design social aux politiques françaises de gestion des risques naturels

cuer une zone, etc. -, elles-mêmes non figées selon : l'ampleur de l'évènement, le lieu où il se produit et le contexte, par essence toujours unique (Ruin et Lutoff 2004). Par exemple, une crue rapide survenant dans un contexte urbain en journée ou en milieu rural de nuit n'aura pas les mêmes conséquences (i.e. en juin 2010 dans le Var, on dénombre 27 morts et disparus dus aux inondations qui se sont produites à l'heure de sortie des bureaux). Les compétences requises pour mettre en œuvre les consignes incluent aussi bien les actions individuelles et collectives à mener que la connaissance des lieux où elles adviennent. Cette double compétence " action et connaissance des lieux » n'est pas innée et nécessite alors d'être communiquée, appropriée et mise en œuvre. Ainsi, des habitants peuvent avoir une bonne connaissance de leur territoire sans nécessairement connaître les consignes de sécurité, tandis que des touristes peuvent les connaître sans savoir comment les appliquer dans un environnement peu familier. La perception d'une situation conduit à tantôt bien évaluer, sous-évaluer ou surévaluer les risques (Kouabenan, Cadet, Hermann et Munoz-Sastre 2006). L'adoption de comportements appropriés est également liée à l'adéquation entre les ressources personnelles et les mesures institutionnelles dont l'individu peut se saisir. Le Haut Comité Français pour la Défense Civile (HCFDC) constate déjà en 2008 que la population est largement tenue à l'écart de l'information sur les dangers, contribuant en cela à la déresponsabiliser et à entretenir la méfiance vis-à-vis des pouvoirs publics. Les individus sont exclus des exercices de sécurité civile alors qu'ils sont les premiers concernés en cas d'évènement. En 2012, le HCFDC note de manière lapidaire que :

L'État a donc du mal à infléchir, dans les faits, la doctrine du citoyen "acteur de sa sécurité". Il souffre de l'ancienne tradition "dormez bien, le gué veille", doctrine très ancrée dans une partie de l'administration publique. (p. 76)

Partant du constat que, d'un côté l'individu est légalement responsable de ses comportements en situation critique et que cela implique des comportements $a d-h o c$, et que d'un autre, les politiques publiques peinent à atteindre leur cible en termes de gestion des risques, il semble pertinent d'envisager un renouvellement de ces approches en partant des individus et non plus des dispositifs institutionnels. Comme le rappelle Gralepois (2012), le risque n'est pas un sujet socialement débattu et de fait, on constate le peu de mobilisation des habitants sur le sujet en dehors de situations particulières de post-crise (par exemple à travers une association de sinistrés) ou de menace spécifique pesant sur leur environnement immédiat (par exemple à travers l'opposition de riverains face à l'implantation d'une infrastructure jugée préjudiciable à leur qualité de vie). Face au constat que le risque majeur n'est pas une préoccupation quotidienne des individus, comment innover et/ou renouveler ces problématiques complexes en partant des individus et de leurs territoires? 


\section{Ocula ${ }^{24}$ \\ PROJEKT}

Vol 21, No 24 (October 2020) • DOI: 10.12977/ocula2020-47

Béatrice Gisclard | L'apport du design social aux politiques françaises de gestion des risques naturels

\subsection{Quelle pertinence de l'innovation sociale dans le contexte des ri-} sques?

La question des risques a peu été abordée sous l'angle de l'innovation sociale, au sens où cette dernière implique une forme d'appropriation par l'échelle individuelle. Pour autant on voit émerger des initiatives qui, pour disparates qu'elles soient, n'en témoignent pas moins d'un réel potentiel de changement de l'approche des risques (Douvinet et al. 2017 ; Gisclard 2017). L'appropriation par les individus des politiques publiques de gestion du risque pensées à un niveau national (Vinet 2007 ; Becerra et Peltier 2009), impose de concentrer l'attention sur les personnes elles-mêmes en lien avec leur territoire. Dans un contexte économique de plus en plus tendu et concurrentiel, l'innovation sociale fait figure de solution " positive », permettant de concilier potentiel de développement et justice sociale (Klein et al. 2014). L'auto-organisation de communautés centrées sur la résolution de problèmes complexes, nés de nos modes de vie contemporains, est un phénomène émergent aux échelles locales particulièrement intéressant. C'est un concept qui n'est pas nouveau, mais qui suscite un regain d'intérêt depuis quelques années. L'innovation sociale est majoritairement identifiée par des finalités de solidarité et concerne les mobilisations d'acteurs qui permettent de poser des problèmes de société d'une façon nouvelle, tout en assurant des services de façon à accroître la qualité de vie des collectivités et des citoyens (Klein et Harrisson 2010). Mais cette terminologie peut assez vite recouvrir des réalités assez diverses, quant à leur nature, les changements visés ou les processus (Cloutier 2003 ; Richez-Battesti et al. 2012). " Tout fait social, tout processus social est spatialisé » (Valette 2003: 30) et le territoire lui-même peut ainsi être considéré comme un milieu « innovateur » (Lacquement et Quéva 2016) car il influence les formes de mise en réseaux des acteurs locaux, entre identité territoriale partagée et relation conflictuelle avec le global (Laville 2014). À une époque mondialisée qui concourt à un aplanissement des différences culturelles, économiques ou politiques, les caractéristiques intrinsèques des territoires empêchent cette homogénéisation et constituent une forme de résistance. C'est d'ailleurs sur ces revendications identitaires et territoriales que s'appuient des collectifs locaux, basés sur un développement local (Pecqueur 2000).

Le décalage actuel entre les investissements financiers, structurels et non structurels, et la faible conscience du risque des individus, appelle à un changement de paradigme (Douvinet et al. 2013 ; Weiss et al. 2011). Ce constat revient à accepter que la population puisse s'auto-organiser dans le temps de la crise. Cela sous-entend également, pour les gestionnaires de crise, d'intégrer de « l'inexplicable » ou de "l'imprévisible » (Dufès 2016). Or, pour les autorités, cette position est difficile à entendre car elle suppose un lâcher-prise qui ne fait pas partie de la culture de la sécurité civile en général et en France en particulier. Diverses expériences d’innovation sociale (les AMAP, les crèches parentales, les ressourceries, etc.) sont autant de formes d'expression qui ouvrent la voie à une telle demande. La possibilité pour un individu ou une communauté de se saisir de sujets problématiques et de les aborder de fa- 


\section{Ocula ${ }^{24}$ \\ PROJEKT}

Vol 21, No 24 (October 2020) • DOI: 10.12977/ocula2020-47

Béatrice Gisclard | L'apport du design social aux politiques françaises de gestion des risques naturels

çon nouvelle, en parallèle des institutions, semble pertinente pour aborder la question de l'appropriation des risques naturels dans le quotidien. Sa prise en compte dans les services publics, à travers l'innovation publique constitue un terreau favorable à l'émergence de solutions moins bureaucratiques, à même de prendre en compte l'échelon individuel de façon effective.

En revanche, si d'un point de vue théorique, l'innovation sociale semble une piste enthousiasmante, de nombreux obstacles perdurent. La perception du risque par les personnes conditionne en partie leurs motivations à l'action. De plus, la conception du rôle de l'État dans la gestion des risques et les infléchissements de ses politiques ne sont pas nécessairement compris et perçus par les destinataires. Parallèlement, se pose la question de l'échelle du changement social qui peut être réel sur une communauté restreinte, mais sans impact au niveau d'une région. Cependant, si l'innovation sociale est une piste originale pour aborder la gestion des risques, comment peut-on imaginer la mettre en place de manière ascendante, c'est-à-dire avec les populations ? Par ses apports en tant que discipline scientifique et ses capacités à faire de l'individu le point de départ de ses démarches, le design social apparaît pertinent pour favoriser cette forme d'innovation.

\subsection{Induire des changement comportementaux en termes de risques par le design}

À l'instar de l'innovation sociale dont l'analyse ne peut faire l'économie des implications politiques qu'elle recèle, le design social par les changements comportementaux et de rapports sociaux qu'il vise à engendrer, est de nature éminemment politique. L'importance de cette visée projectuelle est perçue par Maldonado (1972 : 54) lorsqu'il suggère que : « la projétation [...] cherche à ouvrir un horizon d'action articulé, cohérent, socialement responsable de l'environnement humain et de son destin ». Le design a pour ambition d'agir sur l'expérience quotidienne au moyen de capacités projectives et créatrices et vise l'amélioration de situations jugées problématiques. C'est ce qu'exprime Simon (1969) : " everyone designs who devises courses of action aimed at changing existing situations into preferred ones » (p.130). Au-delà de ses capacités créatives, ce qui fonde le «designerly way of thinking » (Findeli 2015; Cross 2001), ce sont des modalités propres de penser le monde et de le questionner, afin de proposer des réponses aux « wicked problems » (Rittel et Webber 1973), ces problématiques issues de la complexité croissante des sociétés contemporaines. Cette complexité nécessite dès lors de s'extraire d'une approche basée sur une logique analytique pour s'insérer dans une approche systémique qui accepte la part d'irréductibilité inhérente à l'humain et intègre l'incertitude comme une donnée du processus. Les "lunettes » du designer (Findeli 2014) ont ceci de commun avec les « lunettes » du citoyen (Le Moigne 2007), qu'elles permettent de regarder le monde dans sa complexité, sous l'angle de l'expérience de manière " plus sagement pragmatique » (Le Moigne 2007). Pour Bason (2012), le design permet d'aborder les problèmes avec une «nouvelle perspective ». Plus encore que ses capacités de réponses, ce sont les 
Béatrice Gisclard | L'apport du design social aux politiques françaises de gestion des risques naturels

aptitudes du designer à poser des questions non attendues dans un système organisationnel rigide, en travaillant par « empathie professionnelle » (Bason 2012), qui caractérise la démarche du design.

Les designers n'ont évidemment pas l'apanage des bonnes idées, mais ainsi que l'exprimait North Whitehead " we think in generality but we live in details » (1926, cité par Hibou $2012: 35)$ : une idée peut paraître séduisante tant qu'elle n'a pas été réalisée, testée, appropriée, bref, qu'elle ne s'est pas heurtée à la pesanteur du réel. En permettant cette concrétisation aussi bien avec des objets qu'avec des services, le design affirme sa singularité. Dès lors, ses champs d'intervention sont larges et il intervient sur tout système, objet ou espace reliant l'individu à son environnement (« de la petite cuillère à la ville $»^{3}$ ). Les difficultés à circonscrire précisément ce qu'est le design ne sont d'ailleurs pas si préjudiciables à la discipline (il en est de même pour l'art ou la science) tant que cette dernière vise " an always present human approach aimed at the creation of the not-yet-existing " (Stolterman 2011). L'intégration du bénéficiaire, condition sine qua non de toute démarche participative du design social, repose sur des stratégies permettant au designer de transposer son projet dans des dispositifs tangibles, appropriables par les individus (des jeux, des objets, des interfaces numériques, etc.). Les méthodologies de codesign à travers des ateliers, ou toute forme de dispositif ouvert telles les design fictions (Kerspern, Lippera et Hary 2017) ne se substituent évidemment pas aux autres démarches méthodologiques issues des sciences humaines, mais l'originalité de ces dispositifs et le prototypage rapide des solutions constituent des apports manifestes. La figure emblématique de "l'usager ", destinataire des dispositifs, évolue : jusqu'alors considéré comme le récepteur passif, car non-expert, non-sachant, il s'immisce dans le processus et devient actif, considéré alors comme un expert en usage. Les apports se situent à deux niveaux : le premier est une meilleure acceptation du changement, l'engagement des contributeurs dans le projet étant un ressort psychologique efficace et valide (Joule et Beauvois 1998) ; le deuxième est la valorisation des capacités individuelles par une approche habilitante - enabling system - (Manzini 2015). Cependant, le recours à la participation du public ne doit pas non plus être considéré comme le sésame valant acceptation sans condition. Le revers de ces dispositifs est que les propositions des participants doivent être réellement intégrées et ne pas servir comme simple élément de langage. Par ailleurs les objectifs de changements comportementaux nécessitent l'implication des personnes non pas uniquement en tant que destinataires finaux du projet de design, mais dès l'amont, dans les phases de conception et tout au long du projet. La Commission Européenne note à cet égard que le design social contribue à l'empowerment (la réappropriation des capacités) des individus à un niveau local, en les associant aux projets destinés à inventer des solutions à leurs problèmes socioéconomiques (BEPA 2014).

3 Cité par J. Le Bouf 25 août 2009 « l'Art appliqué à l'industrie » (En ligne, consulté le 29 septembre 2019) : <http://blogs.lecolededesign.com/ designethistoires/2009/08/25/lart-applique-a-lindustrie/>. 
Le design social partage avec l'innovation sociale ce que Richez-Battesti et al. (2012) nomment la dimension "d'expérimentation sociale ». En faisant des allers-retours entre hypothèses théoriques et expérimentations-terrain tout en procédant par itération (en acceptant l'erreur), on met de côté de façon temporaire les contraintes normatives et institutionnelles auxquelles est soumise l'action publique. L'implication effective des individus par la co-conception de solutions tend à augmenter les capacitations individuelles et par là, tend également à modifier les comportements. Dans ce sens, les compétences des designers permettent d'aborder sous un angle nouveau la conception "d'objets » habituellement conçus par des experts (ingénieurs, administrateurs, gestionnaires, etc.) formés à des méthodes déductives, linéaires, séquentielles, analytiques où théorie et pratique sont le plus souvent distinguées, voire complètement séparées. Ces constats autorisent dès lors à penser que le sujet des risques naturels pourrait légitimement être abordé selon ces approches, c'est-à-dire en envisageant des formes de participation des habitants à la gestion des risques à travers le design social.

\section{Expérimenter le risque inondation à travers un atelier créatif}

\subsection{Impliquer les habitants par un atelier créatif sur les territoires du risque}

L'implication des habitants dans des démarches concertées sur leur territoire est un moteur indispensable à une meilleure appropriation de la question des risques dans leur quotidien :

Soit on se dit ben on y va, tout le monde est impliqué et on fait des exercices, qui passent soit par des plates-formes de simulation en situation, soit des exercices d'alerte, deux fois par an, j'en sais rien, où le citoyen il est directement impliqué avec des retours sur les exercices. (Entretien 4/10/14-1) ${ }^{4}$

Cependant ce constat, s'il semble largement partagé par les gestionnaires, pose question quant à la mise en place de dispositifs idoines (Agamben 2007), propices à valoriser cette implication individuelle. Les dispositifs existants sont difficilement duplicables, liés à un territoire par essence unique, dépendants des réseaux d'acteurs locaux et portés par des collectifs au sein desquels agissent des acteurs singuliers. Mais l'innovation sociale ne se décrète pas, pas plus qu'elle ne s'impose sur simple décision institutionnelle.

En s'appuyant sur les résultats issus d'entretiens non directifs $(\mathrm{N}=69)$ et de questionnaires $(\mathrm{N}=689)$ dans le contexte des crues rapides dans le sud de la France, un atelier créatif a été conçu et mis en œuvre afin de tester in situ et concrètement les potentiels perçus d'une forme d'innovation sociale. Impliquer les habitants permet concrètement de se confronter à une pratique et de dépasser les aspects théoriques. Cet atelier s'est déroulé dans la commune rurale de Sauve (Gard, France) et les outils de design ont été co-conçus avec

4 Extrait d'un entretien mené avec un gestionnaire du risque. 
Vol 21, No 24 (October 2020) • DOI: 10.12977/ocula2020-47

Béatrice Gisclard | L'apport du design social aux politiques françaises de gestion des risques naturels

l'agence de design social La Bobine. ${ }^{5}$ Chaque dispositif avait pour objectif de travailler sur ce que le philosophe Jean-Marie Schaeffer (2005) appelle « la feintise ludique », qui permet de mettre les personnes en situation d'acteurs. L'atelier, nommé les Veilleurs de Crues, a été réalisé avec des habitants de la commune qui se sont portés volontaires. Ce risque est familier des locaux car les inondations ont régulièrement impacté le village à travers l'histoire. Le petit fleuve côtier (Vidourle) qui le traverse est connu pour ses crues rapides appelées «Vidourlades », conséquences de pluies cévenoles extrêmement violentes. Le déroulé s'organisait en deux temps : « Sauve en 2030 » et « les $24 \mathrm{~h}$ de la crue ». Les joueurs étaient invités à imaginer les actions qui permettraient aux habitants de Sauve d'être moins vulnérables à une inondation qui survenait en 2030 sur la commune.

Au cours de la première partie, cinq articles de journaux fictifs étaient présentés. Ils relataient des catastrophes advenues en 2030 dans les territoires du Gard et s'inspiraient de thématiques saillantes issues des entretiens : la prévention météorologique, la rupture de barrage, le système assurantiel lié aux catastrophes naturelles (CatNat), la mémoire sociale des évènements et enfin les déplacements en contexte de crue (Image 1).

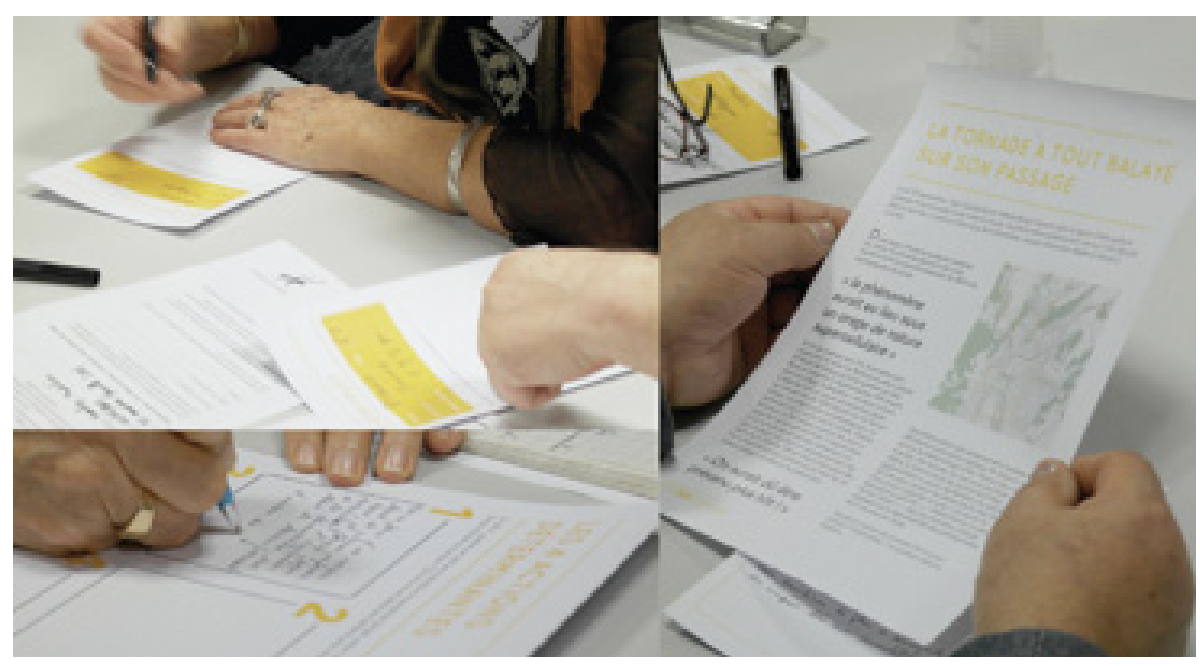

Image 1. Sauve en 2030.

Les groupes devaient en sélectionner un parmi les cinq et expliciter leur choix. Il fallait d'abord lire les cinq articles, en débattre au sein du groupe puis justifier le choix collectif. Une fiche «Zoom sur 3 villes » était remise à chaque groupe afin qu'il détaille, en fonction du thème de l'article, la façon dont des communes avaient dû faire face à la menace grâce aux initiatives des habitants. Parmi les trois villes, deux étaient fictives et la troisième était Sauve. L'objectif était de décrire les actions et stratégies déployées par les habitants ayant permis d'éviter ou de minimiser le risque. La mise à distance temporelle, et le fait

$5<$ http://labobine.co/lesveilleursdecrues $>$. 
Vol 21, No 24 (October 2020) • DOI: 10.12977/ocula2020-47

Béatrice Gisclard | L'apport du design social aux politiques françaises de gestion des risques naturels

que Sauve n'était pas la seule commune concernée, permettait de développer différentes options sans trop se focaliser sur les liens avec l'époque actuelle. En effet, la question des risques reste un sujet sensible dans les politiques de gestion en local et cristallise les tensions entre populations et élus. Les éléments étaient synthétisés sur une carte « les 4 actions déterminantes ». Il était demandé de préciser les actions entreprises par les habitants afin d'éviter la menace dans leur commune. Parmi ces articles de presse, celui ayant focalisé la plus grande attention traitait du risque de rupture de barrage, renvoyant à la présence de deux barrages écrêteurs de crues, situés en amont de Sauve.

La deuxième partie, "les $24 \mathrm{~h}$ de la crue », consistait pour les équipes à décrire leurs actions individuelles et collectives au cours des $24 \mathrm{~h}$ d'une crue intense survenue également en 2030 à Sauve. Cette partie était matérialisée par un jeu de plateau, avec des cartes à jouer et une règle donnée. Chaque équipe piochait au départ des cartes leur indiquant un contexte particulier (une personne à mobilité réduite, un jour férié, etc.), un moment (la nuit, le matin, etc.) ainsi que l'état des réseaux de communication (hors réseau, réseau disponible, etc.). Un support au format A3 était remis à chaque équipe, représentant un cercle découpé en 24 fuseaux qui matérialisait les $24 \mathrm{~h}$. Selon les heures, les équipes étaient invitées à expliquer de manière spatiale et temporelle comment se sont passées les $24 \mathrm{~h}$ d'une Vidourlade et à décrire ce qu'ils avaient fait individuellement et en équipe. Les cartes piochées étant uniques, chaque équipe partait avec une situation différente. Ce dispositif permettait de jouer sur des contextes de crues différents afin de susciter des réponses variées (Image 2).

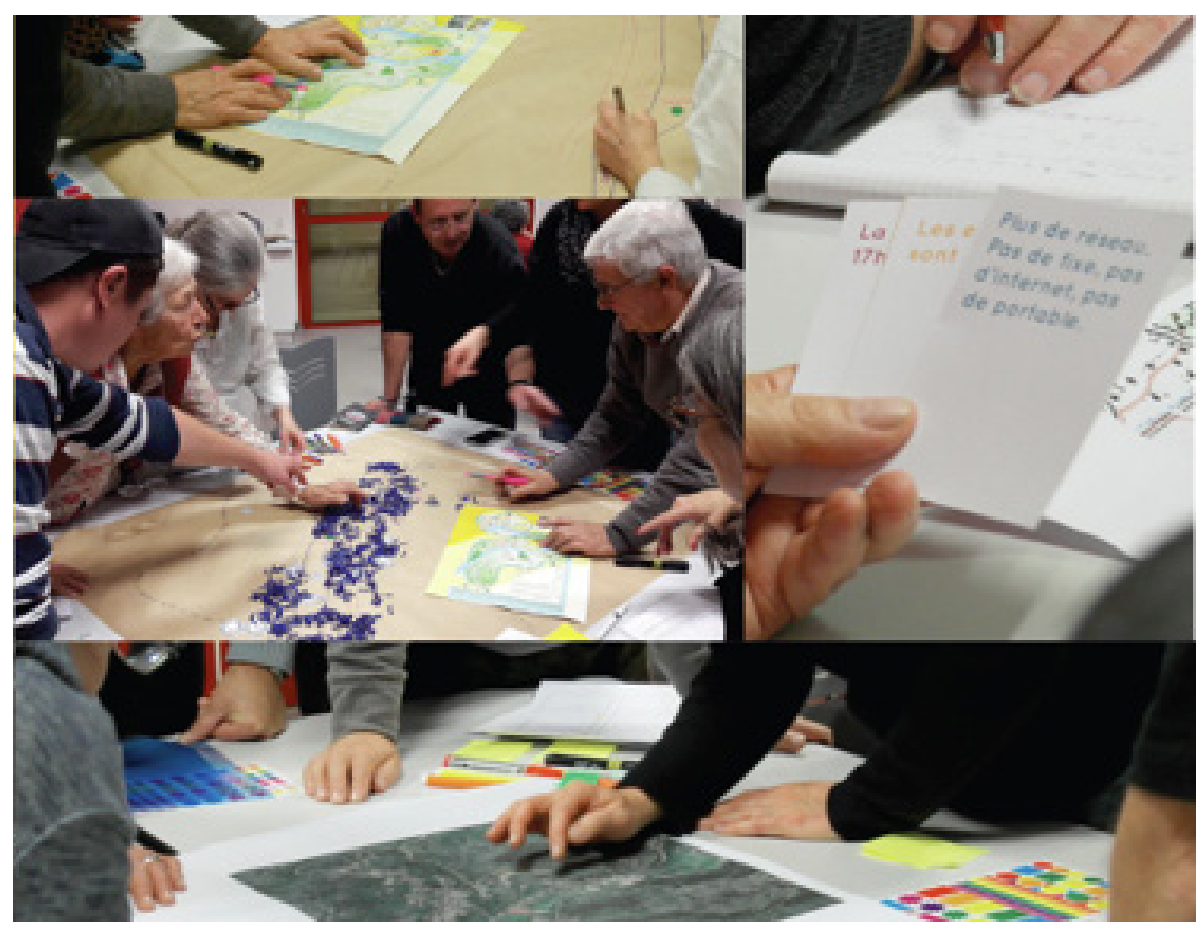

Image 2. Les $24 \mathrm{~h}$ de la crue. 
Vol 21, No 24 (October 2020) • DOI: 10.12977/ocula2020-47

Béatrice Gisclard | L'apport du design social aux politiques françaises de gestion des risques naturels

Bien que le scénario de la crue soit situé dans le futur, ces cartes donnaient un cadre réaliste aux actions sans brider l'imagination. De là, les participants devaient matérialiser leurs déplacements et leurs comportements à partir de supports divers. Deux équipes disposaient de cartographies précises de la commune, l'une de type vue aérienne de type Google Earth@ et l'autre de type SCAN 25® de l'IGN, ${ }^{6}$ les deux autres ayant un support vierge permettant de dessiner, une de ces deux équipes ayant néanmoins à disposition la carte fournie par l'Office du Tourisme et bien connue des habitants. Ils devaient représenter l'emprise de la crue soit avec des papiers bleus préalablement découpés, soit avec les objets qu'ils avaient à disposition. Le choix de ces petits papiers s'est imposé pour permettre aux participants de manipuler le plus simplement possible des objets matérialisant la surface inondée et de revenir dessus autant de fois qu'ils le souhaitaient. La représentation du territoire par la cartographie constitue un élément important dans la construction du risque. Les zones inondables n'étaient volontairement pas indiquées et c'était aux joueurs de les déterminer selon leurs souvenirs des crues passées. Du matériel était mis à la disposition des joueurs (matériel de dessin, feutres, gommettes, papiers colorés, ciseaux, scotch, etc.) ainsi que des petites figures papier symbolisant des personnages, des animaux, des objets de la vie quotidienne (électroménager, télévision, mobiliers) (Image 3).

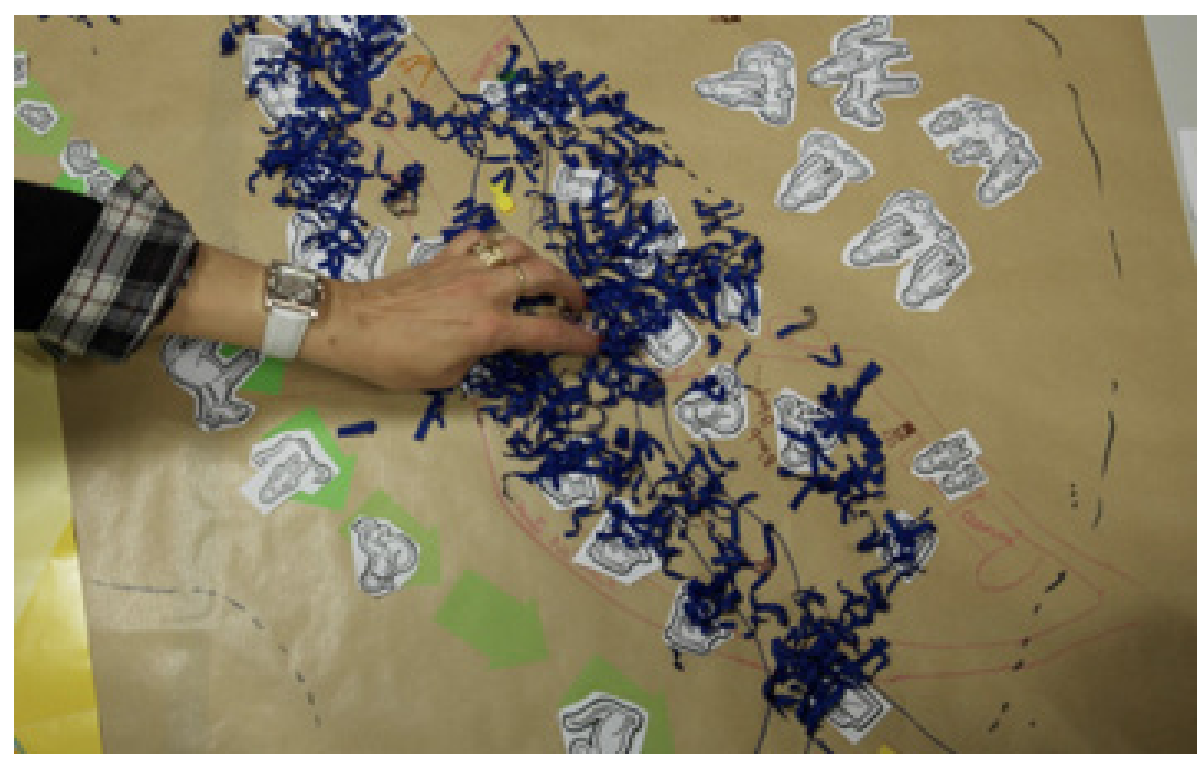

Image 3. Éléments symboliques du quotidien.

Ces éléments additionnels étaient une invitation envers ceux qui ne se sentaient pas à l'aise dans le dessin pour exprimer leurs faits et gestes dans l'action. L'atelier se clôturait sur l'élection du scénario qui apparaissait comme

6 Institut National de l'Information Géographique et Forestière : SCAN 25 (En ligne, consulté le 29 septembre 2019) : <http://professionnels.ign.fr/index.html>. 


\section{Ocula ${ }^{24}$ \\ PROJEKT}

Vol 21, No 24 (October 2020) • DOI: 10.12977/ocula2020-47

Béatrice Gisclard | L'apport du design social aux politiques françaises de gestion des risques naturels

le plus pertinent aux yeux des participants et la mise en discussion de certains points comme les barrages (sources de rumeurs et de controverses) et la compétence Gestion des Milieux Aquatiques et Prévention des Inondations (GEMAPI 2015),7 jusqu'alors ignorée des habitants et sur laquelle ils se sont montrés particulièrement attentifs.

\subsection{Quels enseignements retenir ?}

Les résultats obtenus les plus significatifs sont la mise en action de collaborations entre sujets par le projet permettant de dépasser fondamentalement le discours, et la capacité de créativité dont ils ont fait preuve par le jeu. Les représentations du risque sur le territoire étaient étudiées par le truchement de supports cartographiques différents et il a été souligné que les représentations des zones inondables étaient variables en fonction des joueurs et des supports. Les résultats tangibles se situent dans la mise en débat de problématiques complexes liées à l'inondation et dans l'approche collective de pratiques généralement cantonnées à la dimension individuelle. L'apport du design a été significatif à différents niveaux : au départ, en raison de la reconnaissance par l'ensemble des participants de la qualité des supports (affiches, cartes, identité visuelle, etc.) ce qui leur a permis une bonne appropriation du contexte, que des outils classiques - discussion orale, papier, crayons - auraient amoindrie. La représentation de leur territoire ainsi que les supports de représentation de leur quotidien ont favorisé leur immersion dans la fiction qui leur était proposée. Cette attention portée à leur présence et à la valeur de leurs propositions a été ressentie comme un encouragement bienveillant et catalyseur. En un deuxième temps, l'apport du design a permis aux habitants de se saisir pleinement de la complexité d'un sujet jusqu'alors réservé aux experts (élus, gestionnaires, État), en les autorisant à intervenir en formulant des scénarii et des solutions à leur échelle. Il les a sorti - temporairement - d'un statut d'acteurs passifs face aux consignes institutionnelles, et les a enjoint à se saisir de la problématique du risque inondation sur leur commune. À travers ce changement de posture, le design contribue à renouveler des stratégies d'intégration des habitants dans la gestion des risques. Et malgré des approximations (ou peut-être, grâce à elles), 16 personnes (sur les 20 participants) ont répondu favorablement à la question " seriez-vous prêt à poursuivre avec les veilleurs de crues ? ». Ces résultats demeurent modestes, mais ils contribuent à la conscience du risque à laquelle ils ouvrent des perspectives collectives. Or la dimension commune et partagée des souvenirs conditionne et construit la mémoire sociale des évènements (Halbwachs 1925).

Les personnes ont fait part de leur plaisir et de leur contentement à participer à l'atelier malgré la gravité du sujet. En dépit des réticences des élus et des gestionnaires à affronter ouvertement des risques encourus avec les populations par crainte de "faire peur », cet atelier a été réellement investi

7 Compétence GEMAPI (En ligne, consulté le 29 septembre 2019) : <http:// www.rhone-mediterranee.eaufrance.fr/gestion/locale/gemapi/loi-decrets.php $>$. 
Béatrice Gisclard | L'apport du design social aux politiques françaises de gestion des risques naturels

et les personnes n'ont montré aucun rejet vis-à-vis de la thématique qu'ils ont abordée ensemble. La dimension ludique est un élément clé de ces situations de codesign, car outre la capacité projectuelle du « et si... » rappelée par Schaefer (2005), jouer est une activité créative (Winnicott 1958). Dans la mise « en jeu », le designer interpelle l'homo ludens décrit par Huizinga (1938/1988), créant un espace de liberté pourtant cadré (un jeu a des règles et un espace défini) où l'imagination se déploie. Lorsque l'on joue, on joue " sérieusement ", c'est-à-dire avec attention et sans désinvolture aucune. Cette dimension ludique doublée d'une mise en situation par le projet sont les apports que permet le design et qui le distinguent très nettement des contributions issues des entretiens ou des focus groups. L'action de l'individu confrontée à celle des autres participants, l'oblige à dépasser le simple énoncé de ses idées en l'engageant dans le « faire », faisant surgir des possibilités nouvelles, mettant en lumière des impasses conceptuelles et obligeant à trouver des modes de coopération avec les autres joueurs. Ainsi, les participants matérialisent les idées imaginées collectivement et les expérimentent, aboutissant à l'idéation. Dans le contexte des inondations (comme dans toute catastrophe d'ailleurs), l'importance du référentiel spatial de l'individu ainsi que ses dimensions cognitives et affectives nécessitent de multiplier les angles de vue et par conséquent les méthodes afin de cerner le plus finement possible ces variables. Le jeu est mené de manière constructive à partir du moment où les protagonistes partagent un « cadre d'expérience » (Goffman 1991), qui leur permet de situer leurs actions aussi bien vis-à-vis des autres que dans un lieu et ainsi ajuster leur propre position dans une visée projectuelle. Le cadre donné par la communauté habitante est signifiant. L'identité collective du groupe se fonde sur une position sociale homogène, une contribution socialement reconnue et une histoire commune. De plus, il n'y a pas d'action collective sans un enjeu qui devient mobilisateur (Bajoit 2003). L'importance d'une certaine unité sociale et collective (ici, le village rural) est à souligner, car « la capacité d'initier des projets de développement dans une communauté dépend fortement du cadre relationnel dans lequel évoluent les acteurs » (Prévost et Sévigné 2010 : 173).

Cet atelier, basé sur des méthodologies de codesign, a permis de favoriser l'implication des habitants et leur appropriation en local du risque inondation. En dépassant les dimensions discursives et les constats inopérants, ce format autorise une mise en situation individuelle et collective, pour agir concrètement et de façon ludique sur le risque. À travers une dimension expérimentale et en procédant par itérations, le collectif s'émancipe des contraintes institutionnelles et normatives. Cet impératif de penser le changement par les individus nécessite des dispositifs innovants auxquels le design social peut ici faire écho de manière pertinente. Il valorise l'expertise individuelle et l'implication effective des individus dans les dispositifs participatifs (et non leur simple présence) et tend à augmenter leurs capacitations (Sen 1992). En mettant en exergue leur créativité, cette implication dans le dispositif induit in fine des changements comportementaux. Ce dispositif a permis de co-concevoir des solutions en jouant, d'initier des réflexions origi- 
nales sur la question du risque de crues en général et à Sauve en particulier en imaginant comment les habitants peuvent intervenir à leur échelle en cas de crue du fleuve.

\section{Un engagement individuel et collectif par le projet}

\subsection{Quelles modalités de partage des responsabilités face au risque?}

La dimension collective d'une démarche participative telle que celle présentée n'est pas la garantie d'une vision juste et bénéfique pour tous : elle est signifiante, mais elle n'est pas pour autant représentative. L'appropriation de la question des risques naturels de manière réellement autonome par les habitants renvoie in fine à l'attribution des responsabilités en termes de sauvegarde des populations. La notion d'empowerment rencontre peu d'écho au niveau individuel dans un état fortement centralisé tel que la France. Les éléments du « contrat » vis-à-vis du risque n'étant pas explicites, les individus ignorent qu'ils sont acteurs de leur sécurité (ou qu'ils peuvent l'être) considérant que l'État-providence à qui ils ont délégué cette fonction y pourvoira ainsi qu'il l'a fait jusqu'à présent. Pour le dire autrement, l'auto-organisation spontanée de collectifs face au risque impliquerait que ces derniers considèrent qu'il existe une carence de l'État en la matière et qu'ils doivent dès lors compter sur leurs propres moyens. Cette perception d'un État faillible viendrait en opposition avec l'image renvoyée depuis longtemps par un système stato-centré, basé sur l'intervention de la puissance publique jusqu'aux plus petites échelles territoriales par le biais de ses représentants (les maires). Dès lors, la participation de l'individu à la gestion du risque est un cheminement long et incertain qui part d'une gestion définie par l'institution incluant çà et là des participations individuelles, à des formes d'accompagnement variées syndicats, communes, organismes - pour tendre vers un idéal d'émancipation citoyenne que constituerait l'innovation sociale.

Plus qu'une hypothétique collaboration entre les parties concernées, la recherche précédemment détaillée vise à ouvrir sur de nouveaux modes de relation entre habitants et gestionnaires à travers une innovation sociale territorialisée. La collaboration est le fait de travailler avec quelqu'un. La coopération signifie " travailler à plusieurs, ensemble dans un même but » (Adam 2012), œuvrer à une ou plusieurs personnes à une œuvre ou une action commune. ${ }^{8}$ Étymologiquement ils ont la même racine latine " cum » signifiant " avec ». La nuance concerne le type d'activité : « labor ${ }^{9}$ est la peine que l'on se donne pour faire quelque chose (cum labor), tandis que « operatio $»^{10}$

8 Centre National de Ressources Textuelles et Lexicales « coopération » : <http:// www.cnrtl.fr/definition/cooperation>.

9 Dictionnaire latin-français Gaffiot (1934) p. 878 : <http://www.lexilogos.com/ latin/gaffiot.php?q=labor $>$.

10 Dictionnaire latin-français Gaffiot (1934) p. 1081: <http://www.lexilogos.com/ latin/gaffiot.php? $\mathrm{p}=1081>$. 


\section{Ocula ${ }^{24}$ \\ PROJEKT}

Vol 21, No 24 (October 2020) • DOI: 10.12977/ocula2020-47

Béatrice Gisclard | L'apport du design social aux politiques françaises de gestion des risques naturels

est le travail, l'ouvrage (cum operatio). Historiquement, la collaboration renvoie à la période de domination des nazis en France durant la Seconde Guerre Mondiale, alors que la coopération est liée aux mouvements coopératifs du XIXe siècle. Selon Henri et Lundgren-Cayrol (1997), la distinction entre les deux démarches se situe au niveau de la responsabilité. Dans un contexte de coopération, celle-ci est partagée par le groupe, entraînant une interdépendance réciproque (chaque personne réalise une partie de la tâche globale), tandis que dans un contexte de collaboration, la responsabilité est individuelle et l'interdépendance est associative (chaque personne réalise la tâche à sa manière). Selon les auteurs, la collaboration exige une capacité à travailler en groupe de façon autonome. La coopération tend à plus valoriser l'apprentissage et la collaboration, l'autonomie. Dès lors la coopération plus que la collaboration, apparait comme congruente et l'orientation interdisciplinaire de ce travail se réfère par ailleurs explicitement au modèle coopératif. Le partage des responsabilités caractérise de fait le partage des rôles. Dans la gestion des risques inondation cette répartition des rôles dévolus à chaque partie a évolué dans le temps comme le rappellent Meschinet de Richemond et Reghezza (2010) : autrefois l'administration royale s'occupait de la gestion des débordements tandis que le particulier avait à charge le ruissellement intense et le risque ne se pensait pas indépendamment du territoire sur lequel il pesait.

\subsection{S'engager par le projet}

«Au commencement était l'action » écrivait Goethe dans Faust, engageant ainsi à explorer le « comment » et non plus seulement le «pourquoi ». C'est par cette capacité à apprendre par essai-erreur, en avançant, que l'être humain n'est pas seulement l'être pensant le projet, mais celui qui l'éprouve par l'action, qui se frotte à l'existence au commencement de sa vie avant même de savoir parler. Se projeter dans le futur est un exercice difficile, il est effectivement plus confortable de reproduire des modèles connus, mais la dimension projectuelle articule le « pourquoi » et le « comment » dans un cheminement itératif qui permet la constitution du projet en soi. La personne s'adapte constamment en fonction de son environnement. On pourra rapprocher cette idée avec les travaux des sociologues Crozier et Friedberg (1977) qui suggèrent que face à une situation d'incertitude, l'individu adopte des stratégies en situation et relativisent l'idée qu'il poursuit des objectifs prédéterminés. C'est également ce que Martin (2012) appelle " la recherche pragmatique d'une riposte dans une situation d'incertitude ». Les travaux des auteurs rendent compte des régulations et des ajustements à une échelle microsociale, définis en tant que systèmes d'action concrets.

Lorsque l'on interpelle les habitants sur la question de l'inondation, en mobilisant des savoirs locaux et en co-construisant par une démarche de design social par exemple, il n'y a pas de résistance ni au sujet ni au fait de s'impliquer dedans. À partir du moment où l'individu peut rattacher une préoccupation environnementale (risque inondation) et des préoccupations quotidiennes 


\section{Ocula ${ }^{24}$ \\ PROJEKT}

Vol 21, No 24 (October 2020) • DOI: 10.12977/ocula2020-47

Béatrice Gisclard | L'apport du design social aux politiques françaises de gestion des risques naturels

(son lieu de vie), il semblerait qu'il puisse se saisir des opportunités qui lui sont alors offertes pour agir concrètement et s'impliquer en développant des capacitations (Sen 1992). Les problèmes environnementaux se situent non pas tant au niveau environnemental (ce qui est pourtant perçu comme tel) qu'au niveau social (Uzzell 1999) et cela implique un changement de perspective aussi bien dans la société que pour l'individu. Les questions complexes et systémiques (comme celles des risques) adressées par le monde globalisé auquel nous appartenons tous désormais, peinent à être saisies à cette échelle globale comme en attestent les tensions et les difficultés rencontrées par les grandes instances internationales de type ONU. L'échelle locale apparait pertinente parce qu'elle est souple (les décisions se prennent rapidement) et agile car elle peut autoriser des reconfigurations en fonction des besoins et des nécessités. Cette innovation sociale territorialisée, encouragée par des ateliers créatifs semble alors une perspective intéressante. Face à l'immobilisme ou le manque de volonté des décideurs sur des sujets les concernant au premier chef, des personnes peuvent se mobiliser de manière individuelle ou collective, sans être nécessairement tournées sur des revendications politiques, mais directement orientées vers l'action en lien avec leur lieu de vie.

\section{Conclusions}

Cette première expérimentation ne s'affranchit nullement des limites soulevées par Niedderer et al. (2016) sur la pérennisation et la montée en généralité des innovations proposées par le design social. Elles rejoignent les interrogations de chercheurs travaillant sur les dispositifs de participation du public qui considèrent que la plupart des démarches restent au stade expérimental (Blondiaux et Fourniau 2011). Comment en effet permettre la transposition d'une problématique locale et pensée en tant que telle à une échelle macro, intégrant des différences contextuelles, conduisant à l'énoncé d'une politique publique ? Comment l'action publique qui en résulte peut faire sens auprès des personnes, sans que ne se diluent les finesses propres à la dimension locale (Tromp et al. 2011) ? De plus, l'accaparement par un groupe de personnes (forcément restreint) comporte le risque de voir les actions de ce public privilégier des intérêts particuliers au détriment des valeurs d’intérêt général et d'équité portée par la puissance publique. A contrario, ne pas se préoccuper de la réception et de l'appropriation des politiques publiques à l'échelle individuelle, condamne à réitérer les mêmes erreurs. Pour rappel, l'État français a investi en moyenne ces dernières années 15 millions d'euros par an dans ses politiques de prévention des risques sans s'assurer de leur appropriation et de leur efficacité en local. Bien qu'il soit plus rassurant de s'appuyer sur des modèles techniques et technologiques pour tenter de maitriser les évènements que de se confronter à la pesanteur du social et de faire confiance à l'individu - par essence insaisissable - il existe un potentiel jusqu'à présent sous-exploité (ou minimisé) chez les habitants, qu'il semblerait pertinent de prendre en compte. L'intégration des habitants dans des dispositifs en lien avec le risque se fait peu à peu mais au coup par coup. On peut citer les démarches artis- 
Vol 21, No 24 (October 2020) • DOI: 10.12977/ocula2020-47

Béatrice Gisclard | L'apport du design social aux politiques françaises de gestion des risques naturels

tiques qui permettent d'appréhender le risque de façon ludique et détournée et dans lesquelles le public participe bien volontiers : à titre d'exemple « Jour Inondable » réalisée à Tours en 2012 et déployée en 2019 dans le cadre du Plan Rhône ${ }^{11}$ est une exploration poétique du risque d'inondation. Compte-tenu des perspectives en termes de dérèglement climatique et de l'importance croissante des enjeux de nos sociétés, cela implique un nécessaire rééquilibrage des rôles entre l'État et les individus. Plus que gérer le risque, nous devons désormais apprendre à gérer l'incertitude, et cette complexité rend nécessaire le développement de recherches-actions qui puissent aborder ces problématiques d'un point de vue systémique opérationnel et pourquoi pas, créatif. Développer les capacitations des habitants par des méthodologies issues du design social et leur redonner les clés sur ce qui auparavant leur échappait pour les engager dans l'action face aux risques, constitue une des pistes possibles dans la future gestion des risques.

À travers ce travail interdisciplinaire, on peut s'interroger sur la place allouée au design dans ce contexte de recherche très éloigné des champs traditionnellement explorés par la discipline. Suffit-il d'être designer pour faire du design ou inversement, peut-on faire du design sans nécessairement être designer ? Est-ce la reconnaissance par un public (quel qu'il soit) qui définit le produit, l'objet, le service etc. comme étant du design ? Ces questionnements ont de longue date été formulés dans le champ de l'art et les réponses variables renvoient au caractère contextuel d'un construit social qui dépend tout autant de l'artiste, de l'œuvre, du public et des interactions multiples qui les régissent. Lorsque l'on exerce en tant que designer hors du monde académique, l'interrogation du « quand est-ce du design » tient parfois à la plus ou moins grande tangibilité des productions (i.e. du design de produit jusqu'au design social par exemple) mais elle taraude rarement le designer qui répond à un commanditaire. En effet, ce dernier en faisant appel à lui, légitime de fait sa pratique et son identité professionnelle. Dans le champ de la recherche où la discipline reste relativement méconnue, la reconnaissance de l'effectivité d'une pratique de design est plus difficile à cerner selon les projets dans lesquels le design est sollicité. Le designer n'est plus exclusivement reconnu en tant que designer mais également en tant que chercheur en sciences humaines et sociales. Cette pratique « augmentée » du design contribue alors à brouiller les frontières car les livrables ne sont plus uniquement des produits (entendus au sens large de productions) de design. Néanmoins, parce qu'il a permis un renouvellement des perspectives dans la gestion du risque inondation à travers un dispositif co-construit, le design a été identifié par les parties-prenantes (habitants, gestionnaires et chercheurs) comme vecteur d'approches innovantes volontairement situées en dehors des cadres officiels.

11 La Folie Kilomètre (En ligne, consulté le 29 septembre 2019) : <http:// lafoliekilometre.org/accueil/travaux/une-nuit/>. 
Béatrice Gisclard | L'apport du design social aux politiques françaises de gestion des risques naturels

\section{Bibliographie}

Adam, Michel

2012 «Les valeurs coopératives face à la crise abyssale de nos sociétés », Projectics/ Proyéctica/Projectique, $\mathrm{n}^{\circ} 11-12$, p. 23-39.

Agamben, Giorgio

2007 Qu'est-ce qu'un dispositif ?, Paris, Rivages.

Bajot, Guy

2003 Le changement social, Paris, Armand Colin.

Bason, Christian

2012 «Leading social design: what does it take ?», Social Space, 1, p.24-29.

Becerra, Sylvie ; Peltier, Anne (dir.)

2009 Risques et environnement : recherches interdisciplinaires sur la vulnérabilité des sociétés, Paris, L’Harmattan.

Blondiaux, Loïc ; Fourniau, Jean-Michel

2011 "Un bilan des recherches sur la participation du public en démocratie : beaucoup de bruit pour rien ? », Participations, 1, p. 8-35.

Bureau of European Policy Advisers (BEPA)

2014 Social Innovation. A decade of changes, Report 144p.

Cloutier, Julie

2003 Qu'est-ce que l'innovation sociale ?, Cahier du CRISES, ETo314.

Cross, Nigel

2001 «Designerly ways of knowing: Design discipline versus design science », Design Issues, $\mathrm{n}^{\circ} 17(3)$, p. 49-55.

Crozier, Michel ; Friedberg, Erhard

1977 L'acteur et le système, Paris, Points Seuil.

Dufès, Eric

2016 « L'auto-organisation de la population en situation de crise : l'agir ensemble ». Perspectives. Les cahiers scientifiques de l'ENSOSP, 15, p. 51-79

Findeli, Alain

2014 "Méthodes de la recherche en design - transmission ou reproduction? » Communication orale (actes non publiés) à la Journée d'étude du 2 octobre 2014, Réseau Design en Recherche, Paris, ENSCI les Ateliers.

2015 « La recherche-projet en design et la question de la question de recherche : essai de clarification conceptuelle », Sciences du Design, 1, p. 50.

Gisclard, Béatrice

2017 L'innovation sociale territorialisée : un levier de réappropriation du risque inondation par les habitants. L'exemple des crues rapides dans les territoires ruraux du Gard et du Vaucluse (France), Thèse de doctorat, Université d'Avignon et des Pays de Vaucluse. 
Béatrice Gisclard | L'apport du design social aux politiques françaises de gestion des risques naturels

Gralepois, Mathilde

2012 Face aux risques d'inondation, Paris, Presses de la rue d'Ulm.

Goffman, Erwing

1973 La mise en scène de la vie quotidienne. 1 : La présentation de soi, Paris, les Éditions de Minuit.

Halbwachs, Maurice

1925/ 1994 Les cadres sociaux de la mémoire, Paris, Albin Michel.

\section{HAUT-COMITÉ FRANÇAIS POUR LA DÉFENSE CIVILE (HCFDC)}

2008 Défense civile 2008. Constat et propositions pour une vision globale de la sécurité. $100 p$.

2012 Risques et menaces exceptionnels, quelle préparation? Rapport d'activité 2011. 142p.

Hibou, Béatrice

2012 La bureaucratisation du monde à l’ère néo-libérale, Paris, La Découverte.

Huizinga, Johan

1938 Homo ludens. Essai sur la fonction sociale du jeu, éd. fr. Paris, Gallimard, 1988.

Jacob, Louis ; Desage, Fabien

2015 «Les aménagements de la participation : des, innovation et controverses sociospatiales ", Lien social et Politiques, 73, p. 3-12.

Joule, Robert-Vincent ; Beauvois, Jean-Léon

1998 La soumission librement consentieParis, Presses Universitaires de France.

Kouabenan, Dongo-Rémi ; Cadet, Bernard ; Hermand, Danièle ; Munoz-Sastre, Maria-Theresa (dir.)

2006 Psychologie du risque. Identifier, évaluer, prévenir,Bruxelles, De Boeck Université.

Kerspern, Bastien ; Lippera, Léa ; Hary, Estelle

2017 «ProtoPolicy, le Design Fiction comme modalité de négociation des transformations sociopolitques », Sciences du Design, 5, p. 101-111.

Klein, Juan-Luis ; Harrison, Denis (dir.)

2010 L'innovation sociale. Émergence et effets sur la transformation des sociétés, Québec, Presses de l'Université du Québec.

Lacquement, Guillaume ; Quéva, Christophe

2016 «Introduction? Innovations sociales et développement des territoires dans les campagnes européennes ", Norois, $\mathrm{n}^{\circ} 241, \mathrm{p} .7-13$.

Laville, Jean-Louis

2014 «Innovation sociale, économie sociale, et solidaire, entrepreunariat social, une mise en perspective historique » in Klein, Laville et Moulaert (dir.), L’innovation sociale, Toulouse, Érès, p. 46-90.

Maldonado, Tomas

1971/1972 Environnement et idéologie. Vers une écologie critique,Paris, Seuil (G. Joppolo, trad.). 
Béatrice Gisclard | L'apport du design social aux politiques françaises de gestion des risques naturels

\author{
Manzini, Ezio
}

2015 Design, when Everybody Designs, an introduction to Design for Social Innovation. Cambridge Massachusetts, MIT Press.

Martin, Dominique

2012 «L'analyse stratégique en perspective », Revue Européenne de Sciences Sociales, 50(2), p. 93-114.

Meschinet de Richemond Nancy ; Reghezza Magali

2010 "La gestion du risque en France : contre ou avec le territoire? ", Annales de Géographie, 673, p. 248-267.

Niedderer, Kristina et al.

2016 « Design for behaviour change as a driver for sustainable innovation : Challenges and opportunities for implementation in the private and public sectors », International Journal of Design, 10(2), p. 67-85.

Pecqueur, Bernard

2000 Le développement local, Paris, Syros, $2^{\mathrm{e}}$ ed.

Prévost, Paul ; Sévigny, Bernard

2010 «Les collectivités apprenantes » in Klein et Harrison (dir.), L’innovation sociale. Émergence et effets sur la transformation des sociétés, Québec, Presses de l’Université du Québec, p. 173-191.

Richez-Battesti, Nadine ; Petrella, Francesca ; Vallade, Delphine

2012 "L'innovation sociale, une notion aux usages pluriels : quels enjeux et défis pour l'analyse ? », Innovations, 38, p. 15-36.

Rittel, Horst W. ; Webber, Melvin M.

1973 «Dilemmas in a general theory of planning », Policy Sciences, nº 4, p. 155-169.

Ruin, Isabelle ; Lutoff, Céline

2004 «Vulnérabilité face aux crues rapides et mobilité des populations en temps de crise », La Houille Blanche, 6, p.114-119.

Schaeffer, Jean-Marie

2005 «Quelles vérités pour quelles fictions ? ", L’Homme, 175-176, p. 19-36.

Sen, Amartya

1992 Repenser l'inégalité, trad.P. Chemla,Paris, Seuil, 2000.

Simon, Herbert A.

1969 The sciences of the artificial, Cambridge Usa, MIT Press.

Tromp, Nynke; Hekkert, Paul ; Verbeek, Peter P.

2011 «Design for socially responsable behavior : a classification of influence based on intended user experience », Design Issues, 27(3), p. 3-19.

Uzzell, David L.

1999 « Education for environmental action in the community : new roles and relationships », Cambridge Journal of Education, n²0, p. 397-413.

Valette, Elodie

2003 Pour une approche géographique de l’innovation sociale. L'exemple des 
Béatrice Gisclard | L'apport du design social aux politiques françaises de gestion des risques naturels

territoires ruraux périurbains de la garrigues nord-montpelliéraine, Thèse de doctorat, Université Paris-Diderot-Paris VII.

Vinet, Freddy

2007 «Approches nationales de la prévention des risques et besoins locaux : le cas de la prévision et de l'alerte aux crues dans le Midi méditerranéen », Géocarrefour, $\mathrm{n}^{\circ} 82 / 1-2$, p. 35-42.

Weiss, Karine ; Girandola, Fabien ; Colbeau-Justin, Ludvina

2011 «Les comportements de protection face au risque naturel : de la résistance à l'engagement », Pratiques Psychologiques, $n^{\circ} 17, p$. 251-262.

Winnicott, Donald W.

1958 “The Capacity to Be Alone”. In The Maturational Processes and the Facilitating Environment, in Winnicott, London, UK, Karnac Books, p. 29-36.

\section{Ressources électroniques}

Douvinet, Johnny ; Pallares, Rémi ; Genre-Grandpierre, Cyrille ; Gralepois, Mathilde ; Rode, Sylvain ; Servain-Courant, Sylvie

2013 «L'information sur les risques majeurs à l'échelle communale. Occurrence et facteurs explicatifs du Dicrim, un outil préventif sous-utilisé » in Cybergeo : European Journal of Geography, 658.

<cybergeo.revues.org/26112> [Consulté le 29 septembre 2019].

Douvinet, Johnny ; Gisclard, Béatrice ; Kouadia, Jules Sekouada ; Saint-Martin, Clotilde ; Martin, Gilles

2017 «Une place pour les technologies smartphones et les Réseaux Sociaux Numériques (RSN) dans les dispositifs institutionnels de l'alerte aux inondations en France? » in Cybergeo : European Journal of Geography, 801 <cybergeo.revues.org/27875> [Consulté le 29 septembre 2019].

Henri, France ; Lundgren-Cayrol, Karin

1997 «Apprentissage collaboratif à distance, téléconférence et télédiscussion. Rapport interne no 3 (version 1.7) », Montréal, Canada, LICEF Teluq. <http://www.teluq.uquebec.ca/tec6385/trousse/A3c.htm> [Consulté le 29 septembre 2019].

Le Moigne, Jean-Louis

2007 "L'expert est aveugle sans les lunettes du citoyen » in l'InterLettre Chemin Faisant - MCX-APC, ${ }^{\circ} 38$ <http://www.intelligence-complexite.org/fileadmin/docs/il38.pdf> [Consulté le 29 septembre 2019].

Stolterman, Erik

2011 «The death of design thinking »

<http://transground.blogspot.fr/2011/o7/death-of-design-thinking.html> [Consulté le 29 septembre 2019].

Béatrice Gisclard Béatrice Gisclard est designer et enseignante-chercheure à l'Université de Nîmes (France). Elle a travaillé comme décoratrice d’intérieur, puis elle a repris ses études avec une maîtrise en psychologie de l'environnement, suivie d'un 


\section{Pcula ${ }^{24}$}

PROJEKT

Vol 21, No 24 (October 2020) • DOI: 10.12977/ocula2020-47

Béatrice Gisclard | L'apport du design social aux politiques françaises de gestion des risques naturels

doctorat en géographie (Université d'Avignon). L’essentiel de ses recherches porte sur la place de l'individu dans les politiques de gestion des risques, ainsi que sur le rôle des réseaux sociaux numériques dans la gestion des situations d'urgence. Son approche interdisciplinaire (géographie, psychologie et design) lui permet d'aborder les interrelations que l'individu entretient avec son environnement. Elle est membre du laboratoire PROJEKT (<http://projekt.unimes.fr >) et co-responsable du master DIS (<https:// dis.unimes.fr >) à l'Université de Nîmes.

Elle est secrétaire générale de l'Alliance France Design, et a travaillé à la rédaction du code de déontologie professionnelle, reconnu par la WDO en 2012. Elle a récemment rejoint le comité de l'European Design Academy (EAD). 